\title{
Avaliação in vitro da eficácia de acaricidas sobre Boophilus microplus (Canestrini, 1887) (Acari: Ixodidae) de bovinos no município de Ilhéus, Bahia, Brasil
}

\author{
In vitro valuation of acaricides efficiency to Boophilus microplus (Canestrini, 1887) (Acari: Ixodidae) \\ from bovines at the region of Ilhéus, Bahia, Brazil
}

\author{
Dunezeu Alves Campos Júnior ${ }^{1}$ Paulo Roberto de Oliveira²
}

\section{RESUMO}

O carrapato Boophilus microplus é a espécie de maior distribuição geográfica e importância econômica para os países produtores de bovinos em áreas tropicais e subtropicais do planeta. No Brasil, a Região Nordeste apresenta algumas áreas mais favoráveis para as infestações do gado. O controle químico ainda é a forma mais eficaz de combate deste ectoparasita, mas o manejo inadequado dos acaricidas tem contribuído com o aparecimento de resistência de populações aos produtos disponíveis no mercado. $O$ município de Ilhéus, na Região Cacaueira da Bahia, vem incrementando sua atividade pecuária em substituição à lavoura cacaueira devastada. Foram realizados 30 testes de imersão in vitro (biocarrapaticidogramas) com fêmeas ingurgitadas de $\boldsymbol{B}$. microplus colhidas em propriedades rurais aleatoriamente selecionadas num grupo de 96 entrevistadas na região. O estudo revelou a existência de resistência ou sensibilidade de populações de B. microplus frente aos carrapaticidas de contato mais utilizados nas propriedades de exploração pecuária da região de Ilhéus. Os quatro carrapaticidas testados apresentaram as seguintes eficiências médias: Amitraz - 30,95\%; Deltametrina - 65,04\%; Cipermetrina / diclorvos - 75,73\%; e Triclorfon / coumaphos / cyfluthrin - 75,13\%.

Palavras-chave: Boophilus microplus, Ixodidae, susceptibilidade a acaricidas, controle de carrapatos.

\section{ABSTRACT}

The tick Boophilus microplus is the species with the greatest geographical distribution and economic importance for the countries situated at tropical and subtropical zones of the planet. In Brazil, the Northwest Region is showed as the most favorable to the cattle infestation. Chemical control is an efficient control method employed, but the inadequate handling of acaricides contributed for to the onset of resistant ticks to the available acaricides in the market. The county of Ilhéus, at the Cocoa's Region from Bahia, has been increasing the cattle activity instead of the devastated cocoa's plantations. Between April and September 2000, in vitro imersion tests were performed with engorged females of $\boldsymbol{B}$. microplus collected from 30 farms randomly chosen in a group of 96 interviewed at the region. The current study showed the existence of resistance or sensibility of the populations of B. microplus to the contact acaricides commonly used at cattle properties of the region of Ilhéus. The four acaricides tested showed the following levels of efficiency: Amidin 30.95\%; Deltamethrin - 65.04\%; Cipermethrin / diclorvos 75.73\%; and Triclorfon / coumaphos / cyfluthrin - 75.13\%.

Key words: Boophilus microplus, Ixodidae, susceptibility to acaricides, tick control.

\section{INTRODUÇÃO}

O carrapato comum dos bovinos, Boophilus microplus (CANESTRINI, 1887) (ACARI, IXODIDAE), é atualmente um dos maiores entraves existentes na pecuária bovina dos países localizados em zonas tropicais e subtropicais do planeta.

No Brasil, além de um rebanho bovino composto de raças taurinas, zebuínas e mestiças diferentemente susceptíveis ao desenvolvimento das fases parasitárias deste carrapato, existem ainda variações climáticas muito favoráveis à sobrevivência e desenvolvimento dos seus estádios não parasitários. Além disso, a economia pecuária é prejudicada pelas condições de desenvolvimento econômico,

${ }^{1}$ Departamento de Ciências Agrárias e Ambientais, Universidade Estadual de Santa Cruz (UESC), Rod. Ilhéus-Itabuna Km 16, Salobrinho, 45650-000, Ilhéus, BA, Brasil. E-mail: dunezeu2@yahoo.com.br

${ }^{2}$ Departamento de Medicina Veterinária Preventiva da Escola de Veterinária da Universidade Federal de Minas Gerais (EV-UFMG), Belo Horizonte, MG, Brasil. 
contribuindo para a endemicidade do carrapato em várias regiões do país (CORDOVÉS, 1997).

Dentre as regiões do país, existem áreas no nordeste que apresentam dificuldades no controle do carrapato (EVANS, 1992), em função da diversidade do uso da terra para a agricultura e pecuária, além das frequentes chuvas e alta umidade relativa. Com as temperaturas médias anuais bem acima do requerido para $\boldsymbol{B}$. microplus, de $17^{\circ} \mathrm{C}$, esta região é considerada a mais favorável para a infestação de carrapatos.

O município de Ilhéus localiza-se na região cacaueira da Bahia, sendo que, na década de 70, já foi a responsável por $64 \%$ do PIB estadual em função dos impostos arrecadados pela produção, beneficiamento e exportação do cacau (SILVA, 1972). Aregião, hoje, enfrenta a maior crise econômica de sua história, com a recente falência dos cacauais devido a uma gama de fatores. Sem dúvida, o mais importante deles foi o aparecimento da doença conhecida como "Vassoura-de-bruxa", causada pelo fungo Crinipellis perniciosa, que praticamente dizimou as plantações de cacau na década de 90 .

Desta maneira, tem surgido uma crescente necessidade de diversificação das atividades produtivas na região de Ilhéus, o que tem propiciado um grande incremento na criação de bovinos de corte e de leite no município. As condições naturais extremamente propícias, aliadas às nuances econômicas, políticas, sociais, culturais, nutricionais e sanitárias da região de Ilhéus permitem a permanência dos carrapatos, impedindo a realização de medidas de controle mais eficazes. Assim, o carrapato B. microplus se constitui também na região cacaueira um dos maiores responsáveis pelos altos custos de produção das fazendas de exploração pecuária.

Outro aspecto que muito favorece as populações de $\boldsymbol{B}$. microplus, nas propriedades da região, principalmente as de produção de leite, é o aumento do grau de sangue europeu do rebanho. Por ser um dos caminhos para a maior produtividade leiteira, ocorre a potencialização do efeito prejudicial do carrapato, pela menor resistência desse tipo de animal, visto que quanto maior o grau de sangue zebu nos animais, menor a população de carrapatos (VILLARES, 1941; LEMOS, 1986; TATCHELL, 1987).

Embora existam alguns métodos alternativos, o controle de $\boldsymbol{B}$. microplus está quase exclusivamente relacionado ao tratamento químico com acaricidas de contato. Segundo NOLAN (1994), a ausência evidente de uma política oficial de controle de B. microplus, no Brasil, leva os pecuaristas a adotarem práticas individuais de combate ao carrapato, sem aplicarem as recomendações baseadas em métodos adequados de manejo dos carrapaticidas.
A resistência tem sido estudada e medida por pesquisadores de todo o mundo e em várias regiões do Brasil, porém na região cacaueira da Bahia ainda não existe um registro de dados que demonstrem a eficácia das principais bases químicas carrapaticidas utilizadas para o controle de $\boldsymbol{B}$. microplus. A importância na detecção da resistência destes carrapatos, frente aos acaricidas comerciais mais utilizados pelos produtores, reside principalmente na escolha de um produto comprovadamente eficaz para o controle deste ectoparasita. Isso levaria a uma diminuição do número de aplicações dos carrapaticidas, diminuindo seus efeitos tóxicos, reduzindo os custos de produção, o grau de espoliação dos animais e a população de $\boldsymbol{B}$. microplus nas pastagens (fase não parasitária), além do impacto ambiental pelo uso de produtos químicos. Por outro lado, a obtenção de um perfil regional do problema de resistência de $\boldsymbol{B}$. microplus frente aos carrapaticidas mais empregados poderia servir de subsídio ao planejamento de medidas conjuntas de controle desse parasita.

Com base na hipótese de que existem populações de $\boldsymbol{B}$. microplus, da região de Ilhéus Bahia, apresentando níveis variáveis de resistência contra os principais grupos carrapaticidas do mercado, o presente trabalho teve como objetivo demonstrar o perfil da sensibilidade apresentada pelas amostras coletadas em Ilhéus e arredores. Para isso, foi utilizada a avaliação in vitro do potencial reprodutivo de fêmeas ingurgitadas, o que levou à determinação da eficácia das quatro bases químicas carrapaticidas mais comercializadas na região.

\section{MATERIAL E MÉTODOS}

O município de Ilhéus localiza-se entre os meridianos $39^{\circ} 00^{\prime}$ e $39^{\circ} 30^{\prime} \mathrm{W}$, e os paralelos $14^{\circ} 20^{\prime} \mathrm{e}$ $15^{\circ} 00^{\prime} \mathrm{S}$, na zona fisiográfica denominada Região Cacaueira da Bahia e Região Econômica Litoral Sul do estado da Bahia. Ocupa uma área de $1.712 \mathrm{~km}^{2}$, limitando-se ao norte com os municípios de Aurelino Leal, Itacaré e Uruçuca, ao sul com o município de Una, a sudoeste com Itabuna e Buerarema, ao oeste com Itajuípe e Coaraci, a noroeste com Itapitanga e ao leste com o Oceano Atlântico. Do ponto de vista dos recursos ambientais, trata-se de uma das áreas mais bem dotadas do estado, caracterizando-se como uma região tropical úmida, onde a ação climática representa um elemento fundamental na definição do quadro natural, configurando a excelência dos tipos de relevo e solos da cobertura vegetal e da rede hidrográfica. Tais atributos indicam potencialidades peculiares que se refletem nos padrões de uso da terra e, conseqüentemente, na economia regional. Apresenta 
características climáticas que a enquadra dentro da zona climática classificada como Af de Köeppen: quente, superúmida, sem estação seca pronunciada, caracterizando-se por uma precipitação média anual de 1.910mm nos últimos dez anos (FARIA FILHO, 2000). A precipitação média mensal dos últimos 10 anos se encontra entre 100 e $200 \mathrm{~mm}$, com médias de umidade relativa nunca inferiores a $83 \%$. A temperatura se apresenta com médias mensais elevadas que variam de $22^{\circ}$ a $25^{\circ} \mathrm{C}$, com média de $23,4^{\circ} \mathrm{C}$.

Antes do início da realização das coletas, um cartaz publicitário foi confeccionado e afixado em locais freqüentados pelos pecuaristas. Um total de 96 propriedades foram visitadas na região de Ilhéus - Bahia, e, a partir desses rebanhos cadastrados, aleatoriamente as coletas foram sendo realizadas, à medida que um número mínimo de teleóginas pode ser encontrado na visita a determinado estábulo, ou então amostras foram encaminhadas ao Laboratório de Parasitologia da Universidade Estadual de Santa Cruz. No total, 30 fazendas foram estudadas, e com o auxílio de um aparelho de GPS (Global Positioning System) e do software MapInfo ${ }^{\circledR}$ pode-se precisar exatamente a localização delas no espaço geográfico pesquisado (Figura 1).

As fêmeas de carrapatos ingurgitadas - com um comprimento igual ou superior a $4 \mathrm{~mm}$ - foram colhidas manualmente, diretamente de bovinos de diversas raças e graus de sangue, naturalmente parasitados em 30 propriedades da região do município de Ilhéus. Os animais estavam a, pelo menos, 21 dias sem nenhum tratamento parasiticida, para que não houvesse interferência nos resultados dos testes de sensibilidade. As teleóginas coletadas foram acondicionadas em vasilhames plásticos, identificados, limpos e aerados, para o transporte até o laboratório. Só foram analisados os materiais das propriedades que ofereceram um número mínimo suficiente de teleóginas, e não excedeu o prazo de 48 horas da realização da coleta.

Foram testadas as 4 bases químicas de maior utilização por parte dos produtores da região, ou seja, os mais comercializados pelas casas agropecuárias locais: um representante do grupo químico das amidinas, o Amitraz a 12,5\%; um piretróide, a Deltametrina a 5,0\%; o terceiro uma associação piretróide-organofosforado, Cipermetrina 5,0\% / diclorvos $45,0 \%$ e por fim a associação química Triclorfon 77,6\% / coumafós 1,0\% / cyfluthrin 1,0\%. As concentrações utilizadas nos testes foram rigorosamente idênticas às recomendadas pelo fabricante, sendo os produtos dosados por micropipetas ou balança analítica, diluídos e acondicionados em balões volumétricos de $1.000 \mathrm{ml}$ exclusivamente destinados a esta finalidade. Para cada ensaio de biocarrapaticidograma foi preparada uma diluição correspondente.

O trabalho de laboratório foi realizado nas instalações da Universidade Estadual de Santa Cruz, localizada à Rodovia Ilhéus Itabuna km 16, município de Ilhéus, Bahia, Brasil (39 12’ 49” W e $14^{\circ} 47^{\prime} 40^{\prime}$ ” S). A metodologia utilizada para a pesquisa de sensibilidade foi, segundo a técnica de DRUMMOND et al. (1973), um teste in vitro por imersão de teleóginas nas formulações comerciais com posterior observação da inibição de ovipostura e eclosão larval em comparação a um grupo controle. A técnica foi modificada apenas no que se refere ao tempo de imersão, alterado de 30 segundos para 5 minutos, como convencionado em workshop de acarologistas realizado em Juiz de Fora em 1997 (Segundo LEITE, em comunicação pessoal).

Apenas as teleóginas colhidas nas últimas 48 horas, que se mostravam em perfeito estado físico, sem alterações morfológicas ou restos de tegumento no aparelho bucal foram utilizadas para os testes de sensibilidade.

Seis grupos homogêneos de 10 teleóginas cada, após lavados em peneira com água corrente, foram secos em papel absorvente macio e, em seguida, pesados em balança analítica (precisão de 0,0001g).

Em seguida, permaneceram imersos, durante 5 minutos, em quatro recipientes plásticos identificados, contendo as diluições recomendadas pelos fabricantes de cada acaricida de contato a ser pesquisado, além de dois grupos controles imersos em água destilada.

Após a imersão, as teleóginas foram secas em papel absorvente para se evitar a proliferação de fungos oportunistas. Foram então acondicionadas em placas de Petri descartáveis de $100 \mathrm{~mm}$, devidamente identificadas e levadas à estufa tipo BOD, onde foram mantidas à temperatura de $27^{\circ} \mathrm{C} \pm 1^{\circ} \mathrm{C}$ e umidade relativa do ar de $85 \% \pm 5 \%$.

A partir do décimo dia de postura, foram devidamente registradas em formulário próprio as principais variáveis da pesquisa: mortalidade das teleóginas, peso das posturas (do $15^{\circ}$ ao $18^{\circ}$ dia de postura), percentual de eclodibilidade (de 25 a 35 dias após a pesagem das massas de ovos), eficiência reprodutiva (ER) e eficiência do produto (EP). Estes índices foram observados para cada lote testado, além do grupo controle que apresentou melhor performance reprodutiva. As variáveis dos biocarrapaticidogramas foram então avaliadas, segundo as equações prescritas por DRUMMOND et al. (1973):

Eficiência Reprodutiva

$\mathrm{ER}=$ peso dos ovos $\times$ \% eclosão x 20000** peso das teleóginas 


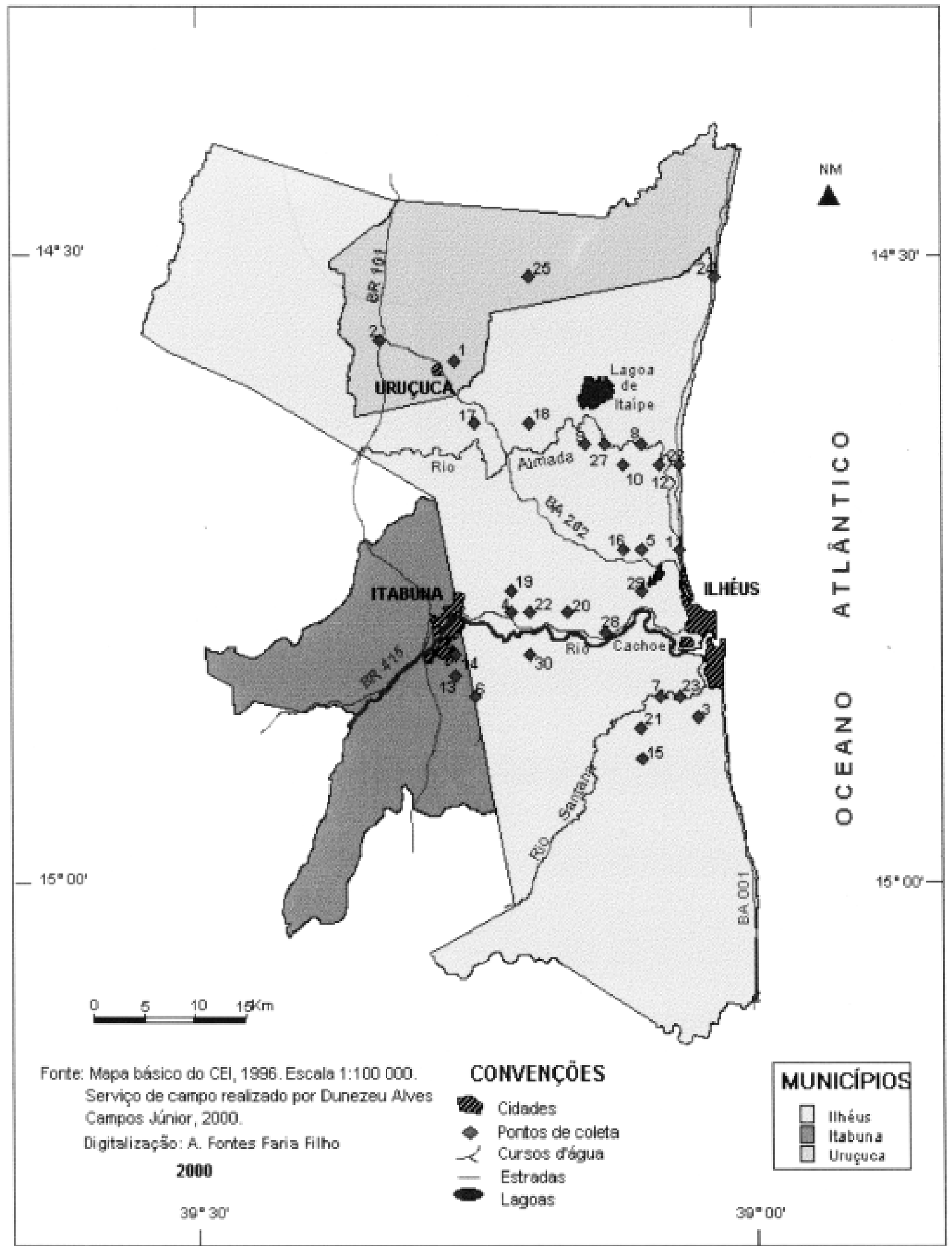

Figura 1 - Localização geográfica das 30 populações de Boophilus microplus testadas na região de Ilhéus - Bahia, no período de abril a setembro de 2000 .

Ciência Rural, v.35, n.6, nov-dez, 2005. 
A constante 20.000 significa o número estimado de ovos em $1 \mathrm{~g}$ de ovos de Boophilus microplus.

Eficiência do Produto

$\mathrm{EP}=\underline{\text { ER grupo controle }- \text { ER grupo tratado }} \times 100$ ER do grupo controle

A análise estatística dos resultados foi executada pelo Teste de Kruskal-Wallis, para a comparação de médias das eficiências dos compostos carrapaticidas testados, com uma significância de 5\% ( $\mathrm{P}>0,05)$. Os gráficos demonstrativos das distribuições de frequência a partir dos percentuais de eficiência em cada população testada foram elaborados através do programa Excel ${ }^{\circledR}$ (Microsoft).

\section{RESULTADOS E DISCUSSÃO}

A eficiência legalmente aceitável para uma base química carrapaticida ser licenciada deve ser igual ou superior a 95,00\% sobre uma cepa sensível de $\boldsymbol{B}$. microplus (MINISTÉRIO DAAGRICULTURA, 1987). Como demonstram as tabelas 1 e 2, todas as quatro bases químicas testadas variaram amplamente nos seus percentuais de eficiência. A menor eficiência observada foi da deltametrina $(0,44 \%)$. A eficiência máxima $(100,00 \%)$ foi observada para o carrapaticida deltametrina e as associações cipermetrina-diclorvos e triclorfon-coumafós-cyfluthrin. O amitraz apresentou eficiência máxima de 98,64\%. Também deve-se observar que, em $40,0 \%$ das propriedades, nenhum dos produtos testados apresentou eficiência considerada aceitável, em $26,7 \%$ delas, apenas um produto se mostrou eficaz e, em 33,3\%, dois produtos obtiveram eficiência superior a 95,00\%. Porém, como podemos confirmar na tabela 3, a formulação de maior eficácia (cipermetrina-diclorvos) apenas alcançou eficiência legalmente aceitável em $43,3 \%$ das propriedades testadas.

Em termos de eficiência média (Tabela 2), o melhor resultado foi o da associação cipermetrinadiclorvos, com 75,73\% de eficiência, que não diferiu estatisticamente $(\mathrm{P}>0,05)$ da mistura triclorfon-coumafóscyfluthrin com $75,13 \%$. O amitraz apresentou a menor taxa de eficiência média nos 30 testes, com 30,95\%.

A baixa sensibilidade de $\boldsymbol{B}$. microplus ao amitraz tem sido notificada, em várias regiões e, por diferentes autores. Porém, a eficiência média de $30,95 \%$ encontrada por este trabalho é a mais baixa entre os valores registrados no Brasil por autores como: ALMEIDA et al. (1994) na BA $(45,7 \%)$; FAUSTINO et al. (1995) em PE (81,94\%); LEITE et al. (1995) em MG; FAUSTINO et al. (1997) em PE
Tabela 1 - Percentuais de eficiência dos produtos carrapaticidas testados em 30 populações de carrapatos Boophilus microplus colhidos em fazendas na região do município de Ilhéus - Bahia no período de abril a setembro de 2000 .

\begin{tabular}{|c|c|c|c|c|}
\hline Teste & $\begin{array}{l}\text { Ami- } \\
\text { traz }\end{array}$ & $\begin{array}{l}\text { Delta- } \\
\text { metrina }\end{array}$ & $\begin{array}{l}\text { Ciper- } \\
\text { metrina/ } \\
\text { diclorvos }\end{array}$ & $\begin{array}{l}\text { Triclorfon/ } \\
\text { coumafós } \\
\text { /cyfluthrin }\end{array}$ \\
\hline 1 & 52,41 & 97,93 & 100,00 & 83,72 \\
\hline 2 & 94,13 & 100,00 & 96,52 & 93,26 \\
\hline 3 & 4,02 & 37,41 & 48,59 & 89,22 \\
\hline 4 & 46,63 & 53,78 & 98,84 & 99,37 \\
\hline 5 & 21,72 & 85,64 & 87,08 & 16,10 \\
\hline 6 & 96,73 & 86,73 & 34,20 & 28,41 \\
\hline 7 & 3,09 & 0,44 & 4,26 & 27,32 \\
\hline 8 & 4,27 & 63,42 & 100,00 & 83,80 \\
\hline 9 & 29,96 & 39,53 & 100,00 & 83,10 \\
\hline 10 & 28,53 & 85,74 & 100,00 & 90,40 \\
\hline 11 & 7,00 & 48,91 & 94,51 & 62,58 \\
\hline 12 & 53,05 & 18,74 & 8,08 & 46,82 \\
\hline 13 & 25,65 & 2,39 & 72,69 & 52,62 \\
\hline 14 & 56,86 & 90,16 & 76,45 & 41,59 \\
\hline 15 & 98,64 & 47,59 & 56,96 & 100,00 \\
\hline 16 & 28,23 & 72,82 & 94,66 & 84,54 \\
\hline 17 & 20,72 & 69,46 & 100,00 & 97,37 \\
\hline 18 & 10,70 & 81,52 & 94,45 & 81,96 \\
\hline 19 & 14,98 & 85,31 & 84,66 & 98,34 \\
\hline 20 & 20,58 & 58,92 & 95,39 & 94,28 \\
\hline 21 & 13,30 & 37,57 & 29,16 & 14,28 \\
\hline 22 & 30,54 & 97,94 & 34,36 & 100,00 \\
\hline 23 & 68,92 & 86,35 & 84,02 & 100,00 \\
\hline 24 & 9,78 & 82,84 & 34,85 & 88,55 \\
\hline 25 & 29,12 & 39,46 & 47,45 & 27,87 \\
\hline 26 & 9,19 & 78,70 & 97,17 & 96,10 \\
\hline 27 & 16,55 & 89,46 & 98,72 & 100,00 \\
\hline 28 & 5,89 & 63,23 & 100,00 & 90,71 \\
\hline 29 & 7,78 & 91,70 & 98,68 & 85,03 \\
\hline 30 & 19,41 & 57,50 & 100,00 & 96,48 \\
\hline
\end{tabular}

(94,48\%); VIEIRA \& TUERLINCK (1997) no RS (91,50\%); OLIVEIRA (1999) em MG (61,16\%) e SANTANA (2000) em PE (40,50\%).

No Brasil, a resistência aos compostos piretróides foi, inicialmente, registrada por LEITE (1988) no RJ, seguido de vários autores a notificar baixas eficiências médias desta base química. A eficiência média encontrada para a deltametrina foi de $65,04 \%$, um valor compatível aos percentuais observados por ALMEIDA et al. (1994) na BA (18,40\%); LEITE et al.(1995) em MG, GO, ES e BA; FAUSTINO et al. (1995) em PE (55,25\%); FAUSTINO et al. (1997) em PE (19,01\%); VIEIRA \& TUERLINCK (1997) na região de Campanha, RS (70,50\%). LEITE et al. (1995) mostraram 
Tabela 2 - Eficiência in vitro de quatro carrapaticidas comerciais testados em 30 populações de carrapatos Boophilus microplus colhidos em fazendas na região do município de Ilhéus - Bahia, no período de abril a setembro de 2000.

\begin{tabular}{|c|c|c|c|c|}
\hline \multirow{2}{*}{ Produtos } & \multirow{2}{*}{ Mínima } & \multirow{2}{*}{ Máxima } & \multicolumn{2}{|c|}{ Eficiência (\%) } \\
\hline & & & Média* & Desvio Padrão \\
\hline Amitraz & 3,09 & 98,64 & $30,95^{c}$ & 28,00 \\
\hline Deltametrina & 0,44 & 100,00 & $65,04^{\mathrm{b}}$ & 27,68 \\
\hline Cipermetrina/diclorvos & 4,26 & 100,00 & $75,73^{\mathrm{a}}$ & 30,62 \\
\hline Triclorfon/coumafós/cyfluthrin & 14,28 & 100,00 & $75,13^{\mathrm{a}}$ & 28,45 \\
\hline
\end{tabular}

* Médias com letras iguais não diferem entre si $(\mathrm{P}>0,05)$

haver uma grande variação na resistência de $\boldsymbol{B}$. microplus frente aos subgrupos de compostos químicos piretróides utilizados como acaricidas.

A associação piretróide-organofosforado (cipermetrina-diclorvos) apresentou nas propriedades estudadas na região de Ilhéus a maior eficiência média dos quatro produtos comerciais testados (75,73\%). Esse valor confirma resultados de OLIVEIRA (1999) em MG (55,47\%) e SANTANA(2000) em PE (89,60\%), que observaram eficiências médias maiores de tais compostos associados, em relação às piretrinas isoladas. Apesar dos organofosforados serem produtos mais antigos e com relatos de resistência em todo o mundo, este grupo químico associado a piretróides pode ser utilizado com eficácia, se um bom manejo carrapaticida for implantado.

A associação triclorfon-coumafós-cyflutrin, apresentou uma eficiência média de 75,13\%. OLIVEIRA (1999) em MG notificou 55,21\% de eficiência média ao coumafós, SANTANA (2000) em PE registrou eficiências médias de $86,44 \%$ e $100,00 \%$ para o coumafós e a associação triclorfon-coumafós, respectivamente.

Entende-se que um simples relato da ineficácia de um determinado acaricida, que não mais atua satisfatoriamente, na maioria das populações de carrapatos, não deve ser o motivo para que o produto não seja mais utilizado em determinada região. Isso

Tabela 3 - Sensibilidade a carrapaticidas de 30 amostras testadas de carrapatos Boophilus microplus oriundos de fazendas da região do município de Ilhéus (BA) cujos resultados apresentaram eficiência $\geq 95,00 \%$.

\begin{tabular}{lc}
\hline Carrapaticidas & Eficiência \\
& $\geq 95 \%$ \\
\hline Amitraz & $6,7 \%(2 / 30)$ \\
Deltametrina & $10,0 \%(3 / 30)$ \\
Cipermetrina/diclorvos & $43,3 \%(11 / 30)$ \\
Triclorfon/coumafós/cyfluthrin & $30,0 \%(9 / 30)$ \\
\hline
\end{tabular}

reforça a necessidade da recomendação dos testes in vitro pelo médico veterinário como forma de diagnóstico individual das propriedades que assiste, além de se constituir um instrumental otimizador da eficiência do controle químico em métodos estratégicos de combate aos carrapatos (ROCHA, 1995).

\section{CONCLUSÕES}

O teste de biocarrapaticidograma deve ser recomendado pelos médicos veterinários da região, visto que se constitui num eficiente e preciso medidor da sensibilidade de $\boldsymbol{B}$. microplus frente aos acaricidas comerciais de contato. Além de indicar o produto mais eficaz para cada população pesquisada, auxilia na descrição do perfil regional dos níveis de sensibilidade e resistência dos carrapatos às bases químicas disponíveis no mercado.

Os resultados dos testes de sensibilidade confirmam a susceptibilidade em níveis variados para as bases químicas testadas, existindo muitas propriedades nas quais nenhum dos compostos tenha apresentado eficiência aceitável. As eficiências médias finais obtidas pelos produtos cipermetrina-diclorvos e triclorfon-coumafós-cyfluthrin demonstram que estes produtos apresentaram níveis aceitáveis frente às populações de $\boldsymbol{B}$. microplus pesquisadas. Em contrapartida, os produtos não associados, constituídos de amidina e deltametrina demonstraram que não mais funcionam na maioria das populações do carrapato testadas.

\section{REFERÊNCIAS}

ALMEIDA, M.A.O. et al. Susceptibilidade do Boophilus microplus a acaricidas na microrregião de Salvador, BA. In: CONGRESSO BRASILEIRO DE MEDICINA VETERINÁRIA, 23., 1994, Olinda, PE. Anais... Olinda, 1994. p.245.

CORDOVÉS, C.O. Carrapato: controle ou erradicação. Porto Alegre: Guaíba Agropecuária, 1997. 197p. 
DRUMMOND, R.O. et al. Boophilus annulatus and Boophilus microplus: laboratory tests for insecticides. Journal of Economic Entomology, v.66, p.130-133, 1973.

EVANS, D.E. Ecologia e controle de carrapatos de bovinos. Juiz de Fora: EMBRAPA-CNPGL, 1992. 32p. (Circular Técnica).

FARIA FILHO, A.F. Zoneamento do meio físico como subsídio ao planejamento agroecológico no município de Ilhéus - Bahia. 2000. 43f. Monografia - Curso de Geografia, Universidade Estadual de Santa Cruz.

FAUSTINO, M.A.G. et al. Eficácia in vitro de produtos carrapaticidas em fêmeas ingurgitadas de cepas de Boophilus microplus da sub-região da Zona da Mata de Pernambuco. Revista Brasileira de Parasitologia Veterinária, v.4, n.2, sup.1, p.58, 1995.

FAUSTINO, M.A.G. et al. Avaliação in vitro da sensibilidade de cepas de Boophilus microplus do estado de Pernambuco a produtos carrapaticidas através de testes de imersão de fêmeas ingurgitadas. Revista Brasileira de Parasitologia Veterinária, v.6, n.2, p.116, 1997.

LEITE, R.C. Boophilus microplus (Canestrini, 1887): susceptibilidade, uso atual e retrospectivo de carrapaticidas em propriedades das regiões fisiográficas da Baixada do Grande Rio e Rio de Janeiro. Uma abordagem epidemiológica. 1988. 151f. Tese (Doutorado em Parasitologia) - Universidade Federal Rural do Rio de Janeiro.

LEITE, R.C. et al. In vitro susceptibility of engorged females from different populations of Boophilus microplus to commercial acaricides. Revista Brasileira de Parasitologia Veterinária, v.4, n.2, p.283-294, 1995.

LEMOS, A.M.A. A resistência genética dos bovinos e o controle do carrapato. Coronel Pacheco: EMBRAPACNPGL, 1986. 42p. (Docs. 6).

MINISTÉRIO DA AGRICULTURA. Normas para registros de parasiticidas de uso pecuário na Brasil. Brasília: Ministério da Agricultura, 1987. 19p. (Mimeografado).
NOLAN, J. Acaricide resistence in the cattle tick Boophilus microplus. In: REPORT OF WORKSHOP LEADER - FAO/ UN consultant, Porto Alegre, RS, Brazil. Abstract... Porto Alegre, 1994. p.21-25.

OLIVEIRA, P.R. Resistência do carrapato Boophilus microplus (Canestrini, 1887) (Acari: Ixodidae) a carrapaticidas em bovinos de leite da região da zona da mata de Minas Gerais. 1999. 33f. Dissertação (Mestrado em Ecologia Animal) - Universidade Federal de Juiz de Fora.

ROCHA, C.M.B.M. Caracterização da percepção dos produtores do município de Divinópolis/MG sobre a importância do carrapato Boophilus microplus e fatores determinantes das formas de combate utilizadas. 1995. 205f. Dissertação (Mestrado em Medicina Veterinária Preventiva) - Universidade Federal de Minas Gerais.

SANTANA, V.L.A. Situação do controle químico do Boophilus microplus (Canestrini, 1887) das subregiões na zona da mata e agreste do estado de Pernambuco, com base em testes in vitro de eficácia de carrapaticidas em fêmeas ingurgitadas. 2000. Dissertação (Mestrado em Medicina Veterinária) - Universidade Federal Rural de Pernambuco.

SILVA, D.A. Possibilidades de aproveitamento agrícola dos solos da região cacaueira da Bahia. Cacau Atualidades, Ilhéus, Ba, v.9 n.1, p.10-14, 1972.

TATCHELL, R.J. Interaction between ticks and their hosts. International Journal of Parasitology, v.17, n.2, p.597605, 1987.

VIEIRA, M.I.; TUERLINCK. Avaliação da resistência do carrapato Boophilus microplus a carrapaticidas em rebanhos de corte e leite do município de Bagé, RS. Revista Brasileira de Parasitologia Veterinária, v.6, n.2, sup.1, p.132, 1997.

VILLARES, J.B. Climatologia Zootécnica. III contribuição ao estudo da resistência e susceptibilidade genética dos bovinos ao Boophilus microplus. Boletim da Indústria Animal, v.4, n.1, p.60, 1941. 